\title{
A Tomada de Decisão em Adultos Jovens e em Adultos Idosos: Um Estudo Comparativo
}

\author{
Decision Making in Young and Older Adults: \\ a Comparative Study
}

La Toma de Decisión en Adultos Jóvenes y en Adultos Mayores: un Estudio Comparativo
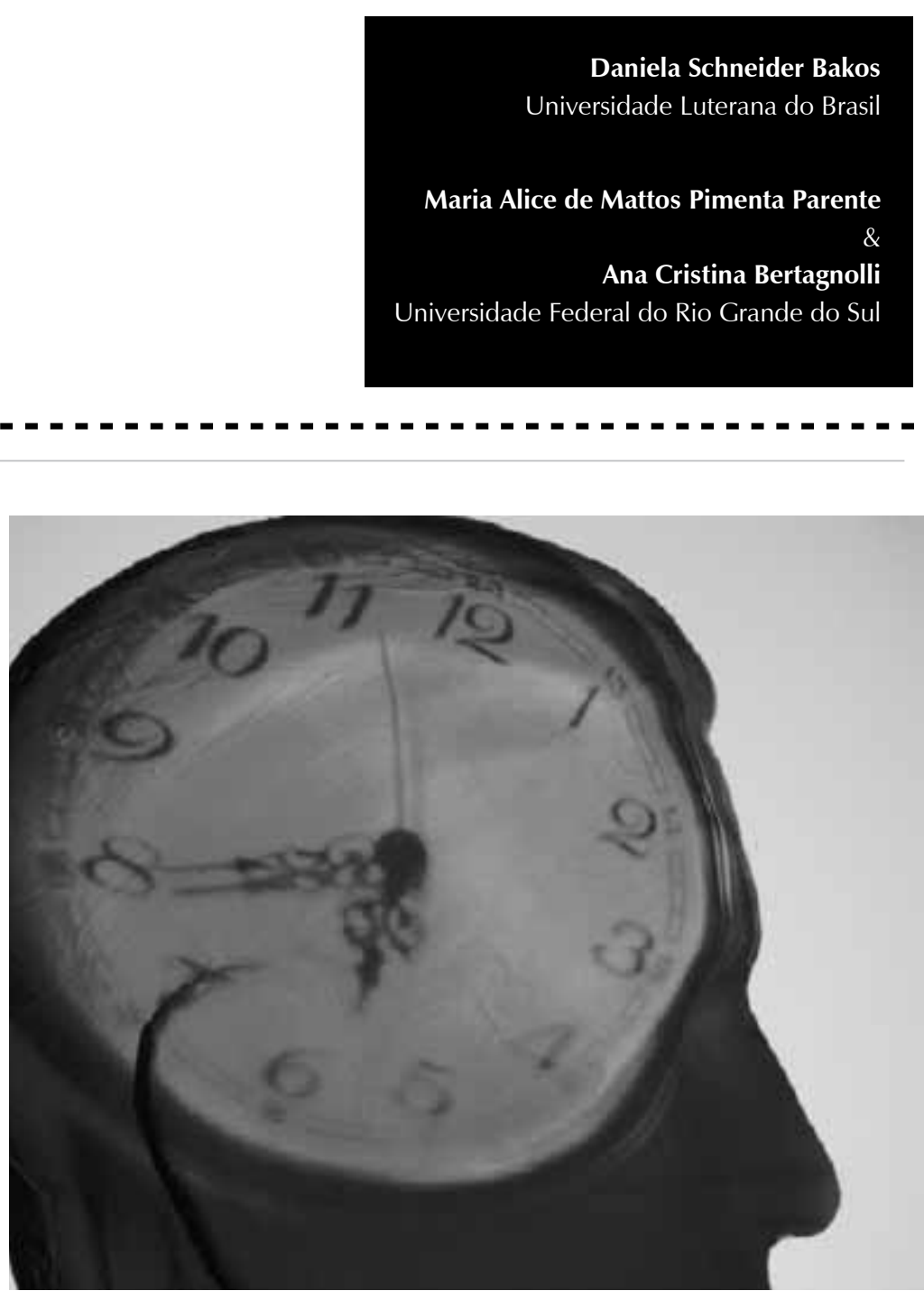
Resumo: O presente estudo comparou adultos jovens e adultos idosos em seu desempenho na IGT. A amostra foi constituída por de 72 indivíduos, distribuídos em dois grupos etários: 36 adultos jovens $(M=29,86, D P=4,63)$ e 36 adultos idosos $(M=66,89, D P=5,19)$. Não houve diferença entre os grupos quanto às escolhas de cartas $(p$ $=0,60$ ), considerando o escore com base no cálculo (mental). Observaram-se, contudo, diferenças significativas no processo de aprendizagem $(p=0,026)$. Considerando o escore com base na frequência (punição), não foram observadas diferenças significativas nas escolhas das cartas $(p=0,48)$ bem como no processo de aprendizagem $(p=0,076)$. Os resultados corroboram estudos anteriores, que minimizam a influência da idade na IGT. Sugerese que a forma de analisar os escores pode estar influenciando o entendimento do comportamento de escolha, juntamente a aspectos referentes à constituição da amostra.

Palavras-chave: Tomada de decisão. Envelhecimento. Processo de aprendizagem. Comportamento de escolha.

Abstract: The present study compared young and elderly adults as to their performance in the IGT. The sample was comprised of 72 individuals, distributed into two age groups: 36 young adults $(M=29.86, S D$ $=4.63)$ and 36 older adults $(M=66.89, S D=5.19)$. Considering the calculation-based score (mental), there was no difference between the groups as the choice of the cards $(p=0.60)$. However, there was significant difference as to the learning processes $(p=0.026)$. There was no significant difference when considering the score based on frequency (punishment), neither as to the choice of cards ( $p=0.48$ ), nor as to the learning process $(p=0.076)$.

The results corroborate previous studies, which do not consider age as a factor that influences IGT performance. The scores analysis might have a strong influence on the understanding of the choice behavior, in addition to aspects relative to sample composition.

Keywords: Decision making. Aging. Learning processes. Choice behavior.

Resumen: El presente estudio comparó adultos jóvenes y adultos mayores en su desempeño en la IGT. La muestra se constituyó por 72 individuos, distribuidos en dos grupos de edad: 36 adultos jóvenes $(M=29,86$, DP $=4,63$ ) y 36 adultos mayores $(M=66,89, D P=5,19)$. No hubo diferencia entre los grupos en cuanto a las elecciones de cartas ( $p=0,60)$, considerando el marcador con base en el cálculo (mental). Se observaron, sin embargo, diferencias significativas en el proceso de aprendizaje $(p=0,026)$. Considerando el marcador con base en la frecuencia (castigo), no fueron observadas diferencias significativas en las elecciones de las cartas $(p=0,48)$ así como en el proceso de aprendizaje $(p=0,076)$. Los resultados corroboran estudios anteriores, que minimizan la influencia de la edad en la IGT. Se sugiere que la forma de analizar los resultados puede estar influenciando el entendimiento del comportamiento de elección, juntamente a aspectos referentes a la constitución de la muestra. Palabras clave: Tomada de decisión. Envejecimiento. Proceso de aprendizaje. Comportamiento de elección.

O processo de envelhecimento, mesmo que saudável, resulta em substanciais mudanças associadas ao lóbulo frontal, especialmente à sua parte mais anterior, o córtex préfrontal (Alvarez \& Emory, 2006; Raz et al., 1997, 2005; Salat, Kaye, \& Janowsky, 2001; Tisserand \& Jolles, 2003; West, 1996, 2000), implicado em processos cognitivos complexos denominados funções executivas (Lezak, 1995; Spreen \& Strauss, 1998; Stuss \& Alexander, 2000). De maneira geral, estudos que enfatizam o funcionamento executivo de indivíduos idosos têm evidenciado declínios sistemáticos dessas funções (Ridderinkhof, Span, \& Van der Molen, 2002; Souchay \& Isingrini, 2004; West, 1996; Zelazo, Craik, \& Booth, 2004), dentre os quais o comportamento de tomar decisões (TD) vem recebendo destaque.
Estudos que enfocam o processo de decisão em diferentes populações têm utilizado como ferramenta de avaliação neuropsicológica a lowa Gambling Task (IGT), um dos poucos instrumentos, até os dias atuais, sensível a déficits na habilidade decisional de pacientes frontais específicos (Bechara, Damasio, Damasio, \& Anderson, 1994). Dentre os estudos internacionais que utilizaram a IGT em idosos, o estudo conduzido por MacPherson, Phillips e Sala (2002) não encontrou diferenças de idade no que tange à tomada de decisão. Os resultados sustentaram influência da idade no desempenho dos indivíduos apenas quando as tarefas dependiam da sub-região pré-frontal dorsolateral, o que sugere que a função de tomada de decisão, conforme mensurada pela IGT, seja menos sensível ao 
envelhecimento saudável. Tais resultados foram corroborados por Lamar e Resnick (2004), reforçando a ideia de uma ausência de diferenças no desempenho de adultos jovens e adultos idosos na IGT.

Objetivando determinar as principais estratégias empregadas por adultos jovens e adultos idosos ao longo de suas escolhas, Wood, Busemeyer, Koling, Cox e Davis (2005) averiguaram o processo de decisão desses dois grupos etários na IGT. O grupo de adultos idosos (idades entre 65 e 88 anos) não diferiu do grupo de adultos jovens (idades entre 18 e 34 anos) no que se refere ao seu desempenho na IGT.

Kovalchik, Camerer, Grether, Plott e Allman (2005), apesar de utilizarem uma versão simplificada da IGT, não verificaram efeito de idade ao comparar grupos de adultos jovens (idades entre 18 e 26 anos) e de adultos idosos (idades entre 70 e 95 anos). Um estudo brasileiro desenvolvido por Schneider e Parente (2006), o qual similarmente almejou examinar o efeito da idade na TD, não encontrou diferença entre os grupos no que tange a sua performance na IGT. Os resultados demonstraram que ambos os grupos selecionaram a mesma frequência de cartas de cada um dos baralhos, o que pode ser sugestivo de uma conduta conservadora.

Já o estudo guiado por Denburg et al. (2005) exibiu uma TD prejudicada em adultos idosos, quando comparados com adultos jovens. Mais especificamente, um subgrupo dos adultos idosos apresentou um déficit na capacidade de decidir vantajosamente, escolhendo um número significativamente maior de cartas dos baralhos desvantajosos. Ao verificar o comportamento decisional em uma nova amostra de indivíduos idosos, Denburg, Reknor, Bechara e Tranel (2006) reproduziram os resultados anteriores, que revelaram prejuízos decisionais em um subgrupo desses participantes. Os resultados vislumbrados por Fein, McGillivray e Finn (2007) e Deakin, Aitken, Robbins e Sahakian (2004) corroboraram os resultados encontrados por Denburg et al. $(2005,2006)$ ao constatarem que adultos idosos saudáveis realizaram escolhas mais desvantajosas em comparação com indivíduos jovens na IGT.

Com base nos estudos apresentados acima, observa-se que o desempenho de idosos na IGT ainda é controverso. Ao se analisar mais detalhadamente os aspectos teóricos e metodológicos dessas investigações, é possível observar fatores que possam estar respondendo, em parte, por tal divergência de dados. Um deles refere-se à inclusão de indivíduos acima de 75 anos de idade, considerados adultos idosos longevos (Baltes, 1998; Singer, Lindberger, \& Baltes, 2003), uma vez que estes podem apresentar uma plasticidade cognitiva diminuída (Singer et al., 2003), o que possivelmente explica o maior prejuízo dentre os adultos idosos. Outro aspecto diz respeito à inserção de adultos abaixo de 20 anos, fase na qual o córtex préfrontal ainda se encontra em desenvolvimento (Nelson \& Luciana, 2001; Pennington, 1998), o que provavelmente atenua as diferenças entre grupos.

As distintas formas de se analisar os escores alcançados na IGT também podem estar colaborando para a diversidade de resultados demonstrada através dos estudos. Enquanto alguns autores analisam diferenças entre grupos a partir da média do desempenho grupal, outros realizam comparações intragrupos, classificando o desempenho de cada indivíduo em prejudicado, limítrofe e não prejudicado. Dessa forma, podem ser constatados subgrupos com padrões de escolha distintos (Denburg et al., 2005). Ademais, os escores atingidos na IGT podem ser interpretados descritivamente, levando-se em consideração as peculiaridades de cada baralho (Bechara, 2007). 
Objetivou-se, na presente investigação, controlar os fatores supracitados, quais sejam, a idade dos indivíduos incluídos na amostra e a maneira de se analisar os escores alcançados, uma vez que estes podem estar contribuindo para a controvérsia hoje existente sobre o comportamento de escolha de adultos idosos. Para tal, um grupo de adultos jovens teve seu desempenho na IGT comparado com indivíduos idosos, sendo os resultados compreendidos através de uma meticulosa análise interpretativa, a partir de sugestões contidas no manual do instrumento, bem como ressaltadas em estudos anteriormente conduzidos. Utilizou-se, ainda, uma nova versão adaptada da IGT, desenvolvida a partir da versão anteriormente adaptada (Schneider \& Parente, 2006), que evidenciava diferenças importantes com relação à original, podendo estas estar influenciando o desempenho dos indivíduos (Schneider, Parente, Wagner, \& Denburg, 2007).

\section{Método}

\section{Participantes}

A amostra foi constituída por de 72 indivíduos de ambos os sexo, da população de Porto Alegre e região metropolitana, distribuídos em dois grupos de acordo com sua faixa etária: 36 adultos jovens (36\% do sexo masculino), com idades entre 25 e 41 anos $(M=29,86, D P=4,63)$ e 36 adultos idosos (16,7\% do sexo masculino) com idades entre 60 e 75 anos $(M=66,89$, $D P=5,19$ ). Foram excluídos da amostra aqueles participantes que apresentavam algum transtorno psicológico, processos demenciais, distúrbios de visão e audição não corrigidos e familiaridade com os testes utilizados. A escolaridade mínima dos participantes aceita para este estudo foi igual ou superior a oito anos completos, o que equivale à conclusão do ensino fundamental.
Através do teste $t$ de Student para amostras independentes, verificou-se que apenas a média de escolaridade diferiu entre os grupos $(t=2,646 ; p=0,01)$, sendo o grupo de adultos jovens mais escolarizado $(M=14,69$, $D P=2,81)$ em comparação com o de adultos idosos $(M=12,75, D P=3,39)$.

\section{Instrumentos e procedimentos}

Questionário de Dados Sociodemográficos: esse questionário foi desenvolvido através de uma adaptação do questionário utilizado pelo Laboratório de Neuropsicolinguística da UFRGS. Teve como objetivo coletar dados de identificação demográfica da amostra bem como aspectos relacionados à saúde, a partir de questionamentos diretos e estruturados.

Mini exame do estado mental (MEEM): o presente instrumento, adaptado para o português brasileiro por Folstein, Folstein e Mchugh (1975) e para a população de Porto Alegre por Chaves e Izquierdo (1992), foi empregado neste estudo no grupo de adultos idosos, com o intento de excluir indivíduos com critérios positivos para quadros demenciais. Foram excluídos idosos que não atingiram 24 pontos (de um total de 30 pontos) nesse instrumento, ponto de corte estipulado para os indivíduos saudáveis com escolaridade superior a 4 anos.

Breve entrevista internacional de neuropsiquiatria modificada (MINI): foi aplicada neste estudo com o intuito de suprimir da amostra indivíduos com algum dos principais transtornos psiquiátricos do eixo I. Trata-se de uma breve entrevista estruturada, segundo critérios diagnósticos encontrados no DSMIV-TR (Manual Diagnóstico e Estatístico dos Transtornos Mentais) e na CID-10 (Código Internacional de Doenças, 10a ed., Sheehan et al., 1998). Com base na validação para a população brasileira conduzida por Amorim 
(2000), foram eliminados os participantes que atenderam os critérios para algum dos transtornos psiquiátricos avaliados.

lowa Gambling Task (IGT): para avaliar a TD, foi utilizada uma nova versão computadorizada da IGT (Bechara et al., 1994), com base na versão adaptada para o português brasileiro por Schneider e Parente (2006). A atual versão diferenciou-se da anterior primordialmente nas instruções iniciais oferecidas aos participantes, que incluíam uma dica quanto à existência de diferenças entre os quatro baralhos. Além disso, a presente versão incluiu um feedback visual, oferecido ao longo do jogo através de uma barra no canto superior da tela (que aumentava na presença de ganhos e diminuía na ocorrência de perdas), o que possibilitou uma ideia dos valores ganhos e perdidos ao longo da tarefa.

Esse instrumento contempla uma situação de tomada de decisão sob incerteza, que envolve escolhas monetárias a curto e a longo prazo, permitindo classificar o comportamento de decisão do indivíduo em termos de habilidade decisional adaptativa ou prejudicada. A tarefa envolve escolhas de cartas de quatro baralhos distintos $(A, B, C$ e $D$ ) ao longo de 100 jogadas (cinco blocos de vinte jogadas cada).

Exemplificando, ao selecionar qualquer carta do baralho $A$ ou $B$, ocorre um rendimento de $R \$ 100,00$. Já ao escolher qualquer carta dos baralhos $C$ ou $D$, um rendimento de $\mathrm{R} \$$ 50,00 é obtido. No entanto, o lucro futuro final de cada baralho varia, uma vez que o valor da punição é maior nos baralhos que pagam quantias mais altas $(A$ e $B$ ) e menor nos baralhos que pagam valores menores ( $C$ e $D)$, isto é, depois de selecionar dez cartas do baralho $A$, os participantes ganham $R \$$ 1000,00, mas também se deparam com cinco punições não previstas ( $R$ \$150,00; $R$ \$200,00; $R \$ 250,00 ; R \$ 300,00$ e $R \$ 350,00$ ), que totalizam uma perda líquida final de $R \$$
250,00. A mesma perda ocorre no baralho $B$, pois, em uma das dez cartas, o participante perde $\mathrm{R} \$ 1.250,00$ de uma só vez. Assim, o baralho $A$ apresenta uma punição mais frequente, embora com valores menores, enquanto no baralho $B$ a punição é menos frequente, mas de maior valor. Os demais baralhos ( $C$ e $D$ ) oferecem um esquema similar de recompensa e punição, mas acarretam lucros líquidos a longo prazo.

\section{Delineamento}

Foi utilizado neste estudo o delineamento quase experimental, do tipo controleexperimental, com pós-teste somente. Os participantes foram selecionados a partir da técnica de amostragem não aleatória de conveniência, em centros de atividades sociais voltados para a terceira idade e em ambientes universitários.

\section{Procedimentos gerais}

A participação dos indivíduos neste estudo foi voluntária, tendo todos os participantes assinado o Termo de Consentimento Livre e Esclarecido. O projeto foi aprovado pelo Comitê de Ética em Pesquisa do Instituto de Psicologia em 15/01/2007, sob o registro no 2006/005. A avaliação foi realizada de forma individual, pelos pesquisadores, em um único encontro de aproximadamente 1 hora e 20 minutos. Essa coleta foi conduzida no Instituto de Pós-Graduação em Psicologia, na UFRGS, ou na residência dos próprios participantes. O MEEM foi aplicado apenas no grupo de adultos idosos, tendo em vista que os indivíduos do grupo de adultos jovens eram, em sua maioria, estudantes universitários que mantinham atividades intelectuais constantes. Todos os instrumentos de caracterização e triagem da amostra foram conduzidos manualmente, ao passo que a IGT foi administrada através do computador. 


\section{Resultados}

\section{Análise dos dados}

A conduta dos indivíduos na IGT foi analisada segundo um breve guia interpretativo, proposto por Bechara (2007). A primeira variável dependente trata do escore líquido total, derivado a partir da subtração do número de cartas retiradas dos baralhos desvantajosos, daquelas retiradas dos baralhos vantajosos: $[(C+D)-(A+B)]$, denominada no presente estudo de escore com base no cálculo (mental). Tendo em vista a ausência de dados normativos para a população brasileira, os participantes da atual amostra tiveram seu desempenho classificado em prejudicados, não prejudicados e limítrofes, conforme pontos de corte a partir de uma distribuição binomial adotada por Denburg et al., (2001, 2005, 2006). No caso de escores acima de +18 , os participantes tiveram seu desempenho considerado não prejudicado, e, entre (-18) e (+18), o desempenho foi classificado como limítrofe, e de (-18) para baixo, prejudicado.

O segundo critério de análise, comumente mencionado na literatura (Bechara, 2007), refere-se ao escore líquido obtido em cada um dos cinco blocos de 20 cartas, ao longo das 100 jogadas. O cálculo empregado para a obtenção desse resultado é o mesmo utilizado para o cálculo do escore com base no cálculo (mental) - $[(C+D)-(A+B)]$ - realizado a cada bloco de 20 cartas, possibilitando verificar o processo de aprendizado.

Considerando as diferentes frequências de punições envolvidas em cada um dos baralhos, optou-se, no presente estudo, por também analisar as escolhas levando em consideração esse critério, isso porque, caso o indivíduo perceba mais nitidamente o aspecto da frequência de punição e recompensa, classificará, em consequência, os baralhos $B$ e $D$ como mais vantajosos, desenvolvendo, possivelmente, marcadores somáticos positivos associados a esses baralhos. Para isso, tanto o escore líquido total quanto o escore líquido por bloco também foi obtido a partir do cálculo $[(B+D)-(A+C)]$, aqui denominado escore com base na frequência (punição).

Ainda é possível efetuar uma análise descritiva dos escores alcançados na IGT através do número de cartas selecionadas em cada baralho. Dentre os baralhos tidos como desvantajosos ( $A$ e $B)$, existem diferenças na frequência e na probabilidade da punição envolvida, tendo o baralho B uma punição muito menos provável por ser esta mais infrequente. Dessa maneira, esse baralho, muitas vezes, não é percebido como tão desvantajoso. O mesmo acontece com o baralho $C$, que, embora seja classificado como vantajoso, pode, em algumas situações, ser compreendido como desvantajoso em função da grande frequência de punições envolvidas (embora estas sejam baixas em magnitudes). Com base nesses aspectos, Bechara (2007) sugere que altas escolhas do baralho $A$ e baixas escolhas do $D$ são fortemente indicativas de uma tomada de decisão prejudicada.

Resultados a partir do escore com base no cálculo (mental)

A partir do teste $t$ de Student para amostras independentes, não foi verificada uma diferença significativa entre os grupos ( $p=$ $0,60)$ com relação ao escore com base no cálculo (mental) $[(A+B)-(C+D)]$. Ambos os grupos selecionaram um pouco mais de cartas dos baralhos $A$ e $B$, e, embora os adultos jovens tenham escolhido ainda mais cartas desses baralhos ( $M=3,5 ; D P=30,45)$ em comparação com os adultos idosos ( $M=$ $0,22 ; D P=22,38)$, essa diferença não se mostrou significativa. 
O processo de aprendizagem foi investigado através de uma análise de variância (ANOVA para medidas repetidas), sendo grupo o fator entre sujeitos e blocos de aprendizagem o fator dentre sujeitos. As variáveis sociodemográficas (escolaridade e gênero) foram avaliadas com relação a sua influência na aprendizagem, e nenhuma delas, além de idade, se mostrou relevante, não sendo, portanto, incluídas no modelo da ANOVA. Os dados evidenciaram diferença significativa entre os grupos, com relação à aprendizagem desenvolvida ao longo da tarefa $(p=0,026)$. $\mathrm{O}$ desempenho dos grupos ao longo dos blocos pode ser contemplado na Figura 1.
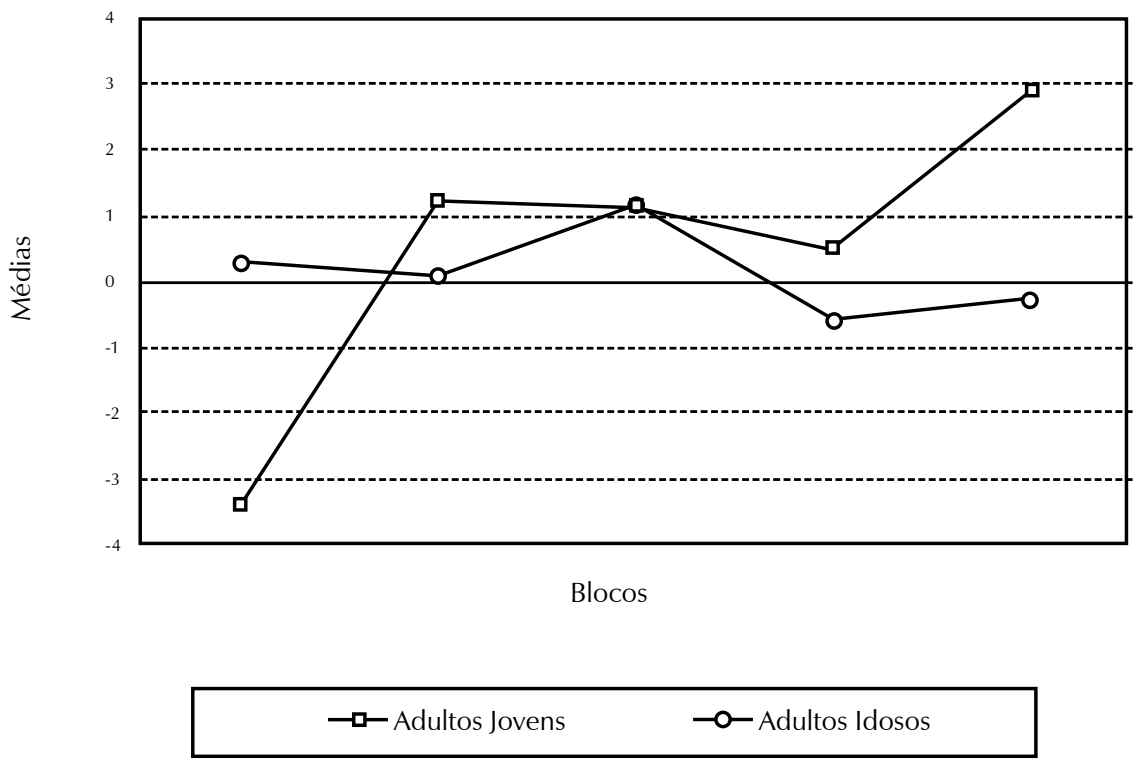

Figura 1. Média de desempenho de adultos jovens e adultos idosos ao longo dos blocos, conforme escore com base no cálculo.

Visando a analisar o desempenho individual de cada um dos participantes em ambos os grupos, calculou-se um escore único para cada participante. A partir do resultado, foram estabelecidos pontos de corte anteriormente mencionados, com base em uma distribuição binomial (Denburg et al., 2001, 2005, 2006), no intuito de classificar o desempenho dos participantes em prejudicados, limítrofes e não prejudicados. Através de um teste Qui-quadrado, não foi verificada diferença entre os grupos quanto ao número de participantes prejudicados $(p=0,216)$.

\section{Resultados a partir do escore com base na frequência (punição)}

A partir do teste $t$ de Student para amostras independentes, não foi verificada uma diferença significativa entre os grupos $(p=0,48)$ com relação ao escore líquido total $[(B+D)-(A+C)]$. Ambos os grupos selecionaram um número maior de cartas dos baralhos $B$ e $D$. Embora os adultos jovens tenham escolhido ainda mais cartas desses baralhos $(M=23,94 ; D P=18,60)$ em comparação com os adultos idosos $(M=20,72 ; D P=19,36)$, essa diferença não se mostrou significativa.

O processo de aprendizagem foi igualmente examinado a partir do escore com base na frequência (punição). Apesar de os dados terem evidenciado uma ausência de diferença entre os grupos ( $p=$ 0,076), é possível observar aspectos distintos na curva de aprendizagem dos dois grupos ao longo das 100 jogadas (Figura 2). 


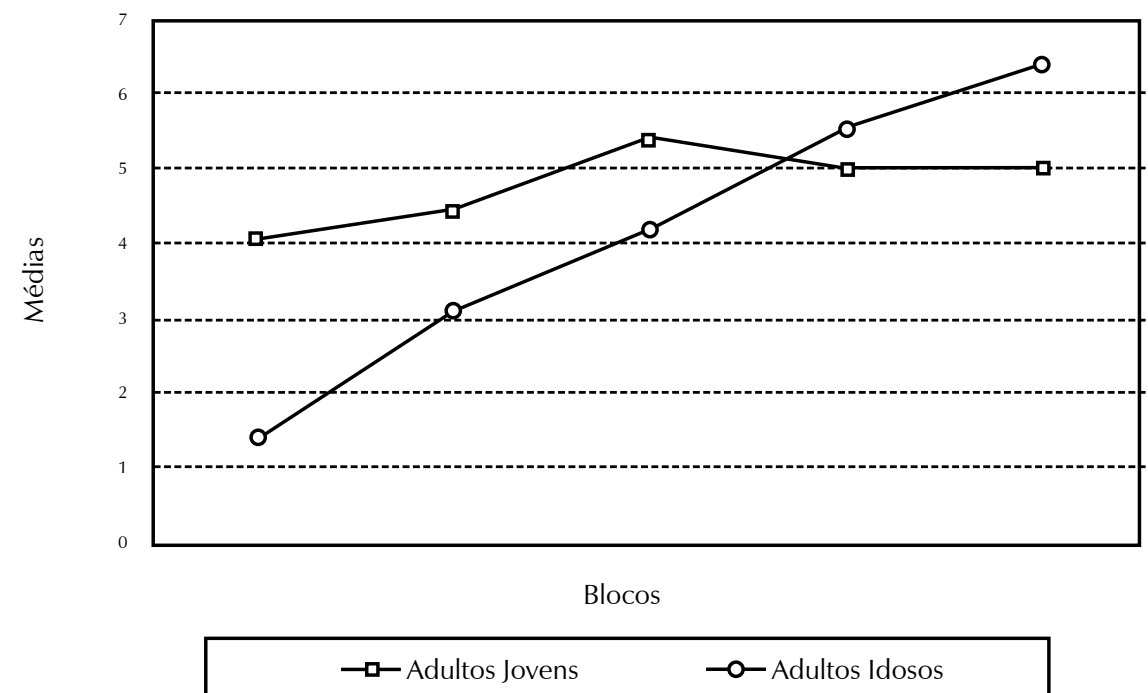

Figura 2. Média de desempenho de adultos jovens e adultos idosos ao longo dos blocos, conforme escore com base na frequência.

O escore único de cada participante foi igualmente classificado conforme distribuição binomial (Denburg et al., 2001, 2005, 2006), no intuito de classificar o desempenho dos participantes em prejudicados, limítrofes e não prejudicados. Através de um teste Qui-quadrado, não foi verificada diferença entre os grupos quanto ao número de participantes prejudicados $(p=0,715)$.

\section{Análise com base nas características individuais de cada baralho}

Com base na sugestão de Bechara (2007) de se analisar individualmente o número de cartas retiradas de cada baralho, observou-se que os baralhos mais escolhidos, em ambos os grupos, foram o $B$ e o $D$, que são baralhos com baixa frequência de punição. Ao se analisar a amostra como um todo, observa-se que os baralhos $A$ e $C$ (que envolvem uma alta frequência de punição) não diferem entre si quanto ao número de cartas escolhidas, entretanto, recebem escolhas estatísticamente inferiores em comparação com as escolhas realizadas nos baralhos $B$ e $D$. Esses dois últimos, por sua vez, são igualmente mais escolhidos, estatisticamente, uma vez que não diferem entre eles, mas entre os outros dois baralhos. Ao se realizar, ainda, uma análise de variância (ANOVA para medidas repetidas) no intuito de verificar a escolha de cartas de cada um dos baralhos ao longo dos cinco blocos de cartas, verificou-se uma diminuição significativa da escolha do baralho $A$ ao longo do tempo $(p<0,001)$, considerando a amostra como um todo.

\section{Discussão e considerações finais}

Os resultados do presente estudo evidenciaram uma ausência de diferença na tomada de decisão de adultos jovens e adultos idosos com relação ao número de cartas retiradas de cada baralho. Contudo, os grupos diferiram significativamente em seu processo de aprendizagem ao se considerar o escore com base no cálculo (mental).

Embora a análise tradicional da IGT classifique a escolha em vantajosa e desvantajosa conforme a preferência pelos baralhos $C$ e $D$ (ganhos e perdas baixas) ou $A$ e $B$ (ganhos e 
Análises segmentadas por baralho, que avaliam a quantidade de escolha de cartas de cada um destes, vêm sendo conduzidas em algumas investigações, as quais também demonstraram maior escolha de cartas dos baralhos B e D por indivíduos saudáveis (Dunn, Dalgleish, \& Lawrence, 2006; MacPherson et al., 2002; Schneider \& Parente, 2006). perdas altos), respectivamente (Bechara et al., 1994), o comportamento da atual amostra demonstrou um padrão distinto, evidenciando uma predileção pelos baralhos $B$ e $D$ (baixas frequências de punição). A diferença entre esse padrão de resultados e aquele normalmente constatado em estudos anteriores pode estar associada à maneira de se analisar os resultados, qual seja, derivar um valor único a partir do somatório do número de cartas retiradas dos baralhos $A$ e $B$ (dupla desvantajosa) e $C$ e $D$ (dupla vantajosa). Esse cálculo pode omitir a formação de outros comportamentos de escolha, uma vez que a obtenção da média impossibilita a mensuração exata da seleção de cartas de cada um dos baralhos separadamente. Isso impede, ainda, que se infira a influência dos distintos aspectos de vantagem e desvantagem (magnitude e frequência das recompensas e punições) envolvidos em cada baralho (Lin, Chiu, Lee, \& Hsieh, 2007).

Análises segmentadas por baralho, que avaliam a quantidade de escolha de cartas de cada um destes, vêm sendo conduzidas em algumas investigações, as quais também demonstraram maior escolha de cartas dos baralhos $B$ e $D$ por indivíduos saudáveis (Dunn, Dalgleish, \& Lawrence, 2006; MacPherson et al., 2002; Schneider \& Parente, 2006). Tomados em conjunto, esses dados têm colocado em pauta o entendimento do baralho $B$ como um baralho desvantajoso, ressaltando a importância de uma análise descritiva dos escores alcançados na IGT (Bechara, 2007; Lin et al., 2007).

Ao se analisar descritivamente o padrão de escolhas da atual amostra, é possível verificar uma progressiva preferência pelas cartas do baralho $A$ ao longo dos cinco blocos. Uma análise de variância (ANOVA para medidas repetidas) evidenciou que o número de cartas retiradas do baralho $A$ apresenta uma gradativa diminuição ao longo dos cinco blocos de cartas. Esse fato faz aventar a hipótese de que um escore mais elevado no que se refere ao escore de aversão ao risco não foi observado por não haver uma diminuição do número de cartas retiradas do baralho $B$. A mesma análise permite que se verifique que as escolhas de cartas dos outros três baralhos $(B, C$ e $D)$, embora tenham diminuído em alguns momentos e aumentado em outros, se mantiveram relativamente constantes ao longo dos cinco blocos.

Conforme sugestão do próprio autor do instrumento (Bechara, 2007), as seleções de cartas do baralho $A$ são fortemente indicativas de uma conduta de busca por risco. Esse autor complementa, ainda, que, ao se considerar os dois baralhos que compõem as escolhas desvantajosas, as opções por cartas do baralho $B$ são menos conclusivas por serem muitas vezes preferidas por indivíduos saudáveis. Nesse sentido, é plausível considerar que as escolhas dos indivíduos da presente amostra refletem uma conduta de aversão ao risco, uma vez que estes foram selecionando gradativamente menos cartas do baralho $A$, apesar de uma escolha constante de um alto número de cartas do baralho $B$.

Ademais, tendo em vista que a alta escolha de cartas do baralho $B$ foi acompanhada por uma seleção similarmente frequente de cartas do baralho $D$, é possível cogitar que a frequência de punição e a recompensa também tenham influenciado o comportamento de decisão dos participantes, isso porque ambos os baralhos apresentam uma baixa frequência de punições em magnitudes altas (baralho $B$ ) e baixas (baralho $D$ ). Visto que esses dois baralhos não diferiram significativamente entre si quanto ao número de cartas retiradas apesar de envolverem diferentes magnitudes, pode-se propor que tenha havido uma preconização do aspecto da frequência dessas recompensas e punições em detrimento dos valores envolvidos. Essa hipótese é fortalecida ao se constatar que os baralhos $B$ e $D$ foram significativamente mais escolhidos, em comparação com os baralhos $A$ e $C$ (ambos com alta frequência de punições, porém com intensidades distintas de recompensa e punição).

No que se refere à ausência de diferença entre os grupos etários quanto ao número de cartas 
retiradas de cada um dos baralhos, esta já havia sido salientada em estudos anteriores (Lamar \& Resnick, 2004; MacPherson et al., 2002; Schneider \& Parente, 2006; Wood et al., 2005). O estudo conduzido por Schneider e Parente, apesar de incluir uma versão diferenciada da tarefa, evidenciou resultados similares ao da atual amostra, não só quanto à questão etária mas também quanto ao padrão de escolha demonstrado.

Apesar disso, outras pesquisas demonstram pior desempenho em adultos idosos, enquanto grupo, em comparação com adultos jovens (Deakin et al., 2004; Denburg et al., 2001, 2005, 2006; Fein et al., 2007). Em uma análise mais aprofundada, Denburg et al. (2001, 2005 e 2006) subdividem a amostra de adultos idosos em subgrupos, conforme o desempenho alcançado na IGT, revelando prejuízo em torno de 33\% desta. Esses dados não podem ser diretamente comparados com os estudos anteriormente mencionados, uma vez que aqueles apresentam a média de desempenho da amostra como um todo, impedindo que se investigue a presença de subgrupos. Inclusive, ao reconduzir as análises do grupo de adultos idosos do estudo de McPherson et al. (2002), Denburg et al. (2005) sugerem que a mesma percentagem de indivíduos com déficit pode ser verificada, ao se realizar uma divisão em subgrupos.

No presente estudo, ao se dividir a amostra em subgrupos, não houve indivíduos prejudicados de acordo com o critério de aversão à frequência. Em contrapartida, ao se considerar o critério de aversão aos valores, houve muitos participantes com desempenho limítrofe. Um escore dentro de um intervalo limítrofe pode ser considerado inconclusivo, uma vez que não difere significativamente de zero, colocando sob suspeita a conduta dos indivíduos segundo esse critério (Denburg et al., 2005). Ademais, ao se condiderar a média dos escores segundo esse mesmo critério, observou-se um desviopadrão muito elevado, o que indica uma grande variabilidade no desempenho dos participantes. É plausível reforçar a ideia, com bases nessas informações e na conduta da amostra como um todo, de que a frequência de ganhos e perdas tenha sido o principal aspecto a guiar as escolhas da atual amostra, uma vez que os escores obtidos segundo esse critério se situaram dentro de intervalos mais conclusivos e apresentaram menor dispersão com relação à média. Em função disso, esses dados não podem ser diretamente comparados com os estudos anteriores, uma vez que aqueles utilizaram apenas o critério de aversão aos valores.

A ausência de diferenças de idade na quantidade de cartas escolhidas em cada baralho, no presente estudo, pode estar associada a explanações distintas, porém relacionadas. Uma delas refere-se a uma ideia sustentada na literatura, a da inexistência de um declínio global nas funções relacionadas ao lóbulo frontal no envelhecimento, a qual sugere que a sub-região frontal seja menos sensível aos déficits que naturalmente acompanham o envelhecimento saudável (MacPherson et al., 2002). Uma segunda explicação diz respeito às características sociodemográficas da amostra, que não incluiu adultos idosos longevos, visto que a literatura se refere a diferenças cognitivas importantes nesse subgrupo em comparação com adultos idosos mais jovens (Baltes \& Smith, 1997). No que se refere à diferença no nível educacional, apesar de os adultos jovens terem apresentado uma escolaridade mais alta, esta, por si só, não pareceu favorecê-los no que tange ao seu desempenho na IGT. Esse fato encontra respaldo nos dados normativos de uma amostra de 932 participantes norteamericanos saudáveis, os quais mostram que os anos de educação responderam por apenas $0,3 \%$ dos 3,6\% de variância entre os escores na IGT (Bechara, 2007).

Um terceiro aspecto aponta o padrão de escolha da atual amostra, uma vez que os participantes empregaram, na maior parte das vezes, processos automáticos de assimilação da freqüência da informação, apoiando-se apenas na informação online. Esse fato, além de contribuir para a definição das preferências de escolha, diminuiu a demanda de recursos da memória de trabalho, contribuindo para 
que não houvesse disparidade entre os grupos. Nesse sentido, estudos comparando diferenças etárias no processamento automático de frequência não têm encontrado desigualdade no desempenho de adultos jovens e adultos idosos (Ellis, Palmer, \& Reeves, 1988; Sanders, Wisc, Liddle, \& Murphy, 1990), o que reforça a ideia de que a frequência de ocorrência é continuamente registrada na memória, independentemente da idade, da educação ou da motivação de um indivíduo (Hasher \& Zacks, 1979, 1984). O estudo realizado por Schneider e Parente (2006) vem ao encontro de tal hipótese, uma vez que seus participantes também selecionaram mais cartas dos baralhos $B$ e $D$, na ausência de diferenças entre grupos.

No que tange à diferença entre os grupos na aprendizagem desenvolvida no decurso da tarefa, esta foi observada quanto ao escore com base no cálculo (mental). Considerando a pressuposição de que a frequência de ganhos e perdas tenha sido o principal aspecto a guiar as escolhas da atual amostra, não é possível tecer uma explicação conclusiva referente ao aprendizado conforme o escore com base no cálculo (mental). Quanto à curva de apredizagem segundo o escore com base na frequência (punição), pode-se verificar que, apesar de haver diferenças na rapidez e na intensidade com que cada grupo desenvolveu a preferência pelos baralhos $B$ e $D$, sendo o grupo de jovens mais rápido na formação de seu padrão de escolha, mantendo-o em uma intensidade constante, esta não se mostrou significativa. Tal desigualdade, embora não significativa, pode ser compreendida por maior rapidez de processamento nos indivíduos jovens, aspecto já referido em estudos anteriores (Walker et al., 1997).

Ao se considerar os resultados do presente estudo, é possível tecer algumas hipóteses explicativas para a atual heterogeneidade de resultados referida na literatura, que mostra tanto a ausência quanto a presença de diferença entre adultos jovens e adultos idosos na IGT. Um aspecto demonstrado no atual estudo refere-se à forma de se analisar os escores obtidos na tarefa, o que pode estar influenciando sobremaneira o entendimento do comportamento de escolha. Além disso, outras variáveis normalmente não controladas, como é o caso da inclusão de indivíduos acima de 75 anos, também podem estar respondendo pela desigualdade demonstrada através dos estudos. É importante salientar, nesse sentido, a necessidade de condução de mais investigações para melhor verificação dos aspectos aventados.

\section{Daniela Schneider Bakos*}

Doutora em Psicologia do Desenvolvimento, Universidade Federal do Rio Grande do Sul, Porto Alegre, RS - Brasil. Psicoterapeuta Cognitivo-Comportamental (Cognitá - Clínica de Terapia Cognitivo-Comportamental). Professora do curso de Psicologia da Universidade Luterana do Brasil, Canoas, RS - Brasil.

Maria Alice de Mattos Pimenta Parente

Doutora em Psicologia. Neuropsicóloga, professora adjunta da Universidade Federal do Rio Grande do Sul, Porto Alegre, RS - Brasil.

E-mail: malicemp@terra.com.br

\section{Ana Cristina Bertagnolli}

Especialista em Psicoterapias Cognitivo-Comportamentais. Psicoterapeuta Cognitivo-Comportamental. Universidade Federal do Rio Grande do Sul, Porto Alegre, RS - Brasil.

E-mail: anacrisbertagnolli@hotmail.com

*Endereço para envio de correspondência:

Rua Mostardeiro, número 5, sala 605 - Bairro Independência - Porto Alegre, RS - Brasil - CEP: 90430-001. E-mail: danigiorgio@hotmail.com 
Referências
Alvarez, J. A., \& Emory, E. (2006). Executive function and the frontal lobe: A meta-analytic review. Neuropsychology Review, $16(1), 17-42$

Amorim, P. (2000). Mini international neuropsychiatric interview (MINI): validação de entrevista breve para diagnóstico de transtornos mentais. Revista Brasileira de Psiquiatria, 22(3), 106-115

Baltes, M. M. (1998). The psychology of the oldest-old: The fourth age. Geriatric Psychiatry, 11(4), 411-415.

Baltes, P. B., \& Smith, J. (1997). A systemic-holistic view of psychological functioning in very old age: Introduction to a collection of articles from the Berlin aging study. Psychology and Aging, 12(3), 395-409.

Bechara, A. (2007). lowa Gambling Task - Professional Manual. Florida: Psychological Assessment Resources.

Bechara, A., Damasio, A. R., Damasio, H., \& Anderson, S. W. (1994). Insensitivity to future consequences following damage to human prefrontal cortex. Cognition, 50(1-3), 7-15.

Chaves, M. L., \& Izquierdo, Y. (1992). Differential diagnosis between dementia and depression: A study of efficiency increment. Acta Neurologica Scandinavia, 85(6), 378-382.

Deakin, J. B., Aitken, M. R. F., Robbins, T. W., \& Sahakian, B. J. (2004). Risk taking during decision-making in normal volunteers changes with age. Journal of the International Neuroscience Society, 10(4), 590-598.

Denburg, N. L., Bechara, A., Cole, C., \& Tranel, D. (2001). lowa Gambling Task performance in elderly persons predicts susceptibility to the influence of misleading advertising. In Annual Meeting of the Society for Neuroscience (p. 27). San Diego, USA.

Denburg, N. Tranel, D. \& Bechara, A. (2005). The ability to decide advantageously declines prematurely in some normal older persons. Neuropsychologia, 43(7), 1099-1106.

Denburg, N. L., Recknor, E. C., Bechara, A., \& Tranel, D. (2006). Psychophysiological anticipation of positive outcomes promotes advantageous decision-making in normal older persons. International Journal of Psychophysiology, 61(1), 19-25.

Dunn, B. D, Dalgleish, T. \& Lawrence, A. D. (2006). The somatic maker hypothesis: A critical evaluation. Neuroscience and Behavioral Review, 30(2), 239-271.

Ellis, N. R., Palmer, R. L., \& Reeves, C. L. (1988). Developmental and intellectual differences in frequency processing. Developmental Psychology, 24(1), 38-45.

Fein, G., McGillivray, S. \& Finn, P. (2007). Older adults make less advantageous decisions than younger adults: Cognitive and psychological correlates. Journal of the International Neuropsychology Society, 13(3), 480-489.

Folstein, M. F, Folstein, S. E. \& Mchugh, P. R. (1975). Mini-mental state. Journal of Psychiatry Resources, 12(3), 189-198.

Hasher, L., \& Zacks, R. T. (1979). Automatic and effortful processe in memory. Journal of Experimental Psychology: General, 108(3), 356-388.

Hasher, L., \& Zacks, R. T. (1984). Automatic processing of fundamental information - The case of frequency of occurrence. American Psychological Association, 39(12), 1372-1388.

Kovalchik, S., Camerer, C., Grether, D., Plott, C., \& Allman, J. M. (2005). Aging and decision making: A comparison between neurologically healthy elderly and young individuals. Journal of Economic Behavior \& Organization, 58, 79-94.

Lamar, M., \& Resnick, S. M. (2004). Aging and prefrontal functions: Dissociating orbitofrontal and dorsolateral abilities. Neurobiology of Aging, 25(4), 553-558.

Lezak, M. (1995). Neuropsychological assessment. New York: Oxford University Press.

Lin, C., Chiu, Y., Lee, P., \& Hsieh, J. (2007). Is deck B a disadvantageous deck in the lowa Gambling Task? Behavioral and Brain Functions, 3(16), 1-10.
MacPherson, S. E., Phillips, L. H., \& Sala, S. D. (2002). Age, executive function, and social decision making: A dorsolateral prefrontal theory of cognitive aging. Psychology and Aging, 17(4), 598-609.

Nelson, C. A., \& Luciana, M. (2001). Handbook of developmental cognitive neuroscience. Cambridge, MA: MIT Press.

Pennington, B. F. (1998). Dimensions of executive functions in normal and abnormal development. In N. A. Krasnegor, G. R. Lyon \& P. S. Goldman-Rakic (Eds.), Development of the prefrontal cortex: Evolution, neurobiology, and behavior (pp. 256-281). Baltimore, MD: Brookes.

Raz, N., Gunning, F. M., Head, D., Dupuis, J. H., McQuain, J., Briggs, S. D., et al. (1997). Selective aging of the human cerebral cortex observed in vivo: Differential vulnerability of the prefrontal gray matter. Cerebral Cortex, 7(3), 268-282.

Raz, N., Lindenberger, U., Rodriguez, K., Kennedy, K. M., Head, D., Williamson, A., et al. (2005). Regional brain changes in aging healthy adults: General trends, individual differences and modifiers. Cerebral Cortex, 15(11), 1676-1689.

Ridderinkhof, K. R., Span, M. M., \& Van der Molen, M. W. (2002). Perseverative behavior and adaptative control in older adults: Performance monitoring, rule induction, and set shifting. Brain and Cognition, 49(3), 381-401.

Salat, D. H., Kaye, J. A., \& Janowsky, J. S. (2001). Selective preservation and degeneration within the prefrontal cortex in aging and Alzheimer disease. Archives of Neurology, 58(9), 1403-1408.

Sanders, R. E., Wisc, J., L., Liddle, C., L., \& Murphy, M. D. (1990). Adult age comparisons in the processing of event frequency information. Psychology and Aging, 5(2), 172-177.

Schneider, D. D. G., \& Parente, M. A. M. P. (2006). O desempenho de adultos jovens e idosos no lowa Gambling Task: um estudo sobre a tomada de decisão. Psicologia: Reflexão e Crítica, 19(3), $442-450$

Schneider, D. D. G., Parente, M. A. M. P., Wagner, G., \& Denburg, N. (2007). lowa Gambling Task: Administration effects in older adults. Dementia \& Neuropsychologia, 1, 66-73.

Sheehan, D. V, Lecrubier, $Y$, Sheehan, K. H, Amorim, P, Janavs, J., Weiller, E., et al. (1998). The mini international neuropsychiatry interview (MINI): The development and validation of a structured diagnostic psychiatric interview for DSM-IV and CID-10. Journal of Clinical Psychiatry, 59(20), 22-33.

Singer, T., Lindenberg, U., \& Baltes, P. B. (2003). Plasticity of memory for the new learning in very old age: A story of major loss? Psychology and Aging, 18(2), 306-317.

Souchay, C., \& Isingrini, M. (2004). Age related differences in metacognitve control: Role of executive functioning. Brain and Cognition, 56(1), 89-99.

Spreen, O., \& Strauss, E. (1998). A compendium of neuropsychological tests - Administration, norms and commentary. New York, USA: Oxford University.

Stuss, D. T., \& Alexander, M. P. (2000). Executive functions and the frontal lobe: A conceptual view. Psychological Research, 63(3-4), 289-298.

Tisserand, D. J., \& Jolles, J. (2003). On the involvement of prefrontal networks in cognitive ageing. Cortex, 39(4-5), 1107-1128.

Walker, N., Fain, W. B., Fisk, A. D., \& McGuire, C. L. (1997). Aging and decision making: Driving-related problem solving. Human Factors, 39(3), 438-44

West, R. L. (1996). An application of prefrontal cortex function theory to cognitive aging. Psychological Bulletin, 120(2), 272-292.

West, R. (2000). In defense of the frontal lobe hypothesis of cognitive aging. Journal of the International Neuropsychological Society, 6(6), 727-729.

Wood, S., Busemeyer, J., Koling, A., Cox, C., \& Davis, H. (2005). Older adults as adaptive decision-makers: Evidence from the lowa Gambling Task. Psychology and Aging, 20(2), 220-225.

Zelazo, P. D., Craik, F. I. M., \& Booth, L. (2004). Executive function across life span. Acta Psychologica, 115(2-3), 167-183. 